(2) Open Access Full Text Article

REVIEW

\title{
Management of thrombotic thrombocytopenic purpura: current perspectives
}

This article was published in the following Dove Press journal:

Journal of Blood Medicine

5 February 2014

Number of times this article has been viewed

\author{
Piers Blombery \\ Marie Scully \\ Department of Haematology, \\ University College London Hospital, \\ London, UK
}

\begin{abstract}
Thrombotic thrombocytopenic purpura (TTP) is a rare, life-threatening thrombotic microangiopathy which causes significant morbidity and mortality unless promptly recognized and treated. The underlying pathogenesis of TTP is a severe deficiency in ADAMTS13 activity, a metalloprotease that cleaves ultralarge von Willebrand factor multimers. This deficiency is either autoantibody mediated (acquired TTP) or due to deleterious mutations in the gene encoding ADAMTS13 (congenital TTP). The elucidation of this disease mechanism has reinforced the rationale and place of current therapies (eg, plasma exchange) as well as providing a basis for the prospective evaluation of immunotherapy with rituximab in addition to classic immunosuppression (eg, corticosteroid) in autoantibody-mediated TTP. This review discusses the current evidence base for therapeutic interventions in acquired and congenital TTP as well as providing a practical approach to the other aspects of investigation and management for which a firm evidence base is lacking. Novel agents that are currently being evaluated in prospective trials and future directions of therapy are also discussed which are expected to make an important contribution to improving outcomes in patients with TTP.
\end{abstract}

Keywords: TTP, rituximab, ADAMTS13

\section{Introduction}

Thrombotic thrombocytopenic purpura (TTP) is an aggressive form of thrombotic microangiopathy which results in multiorgan dysfunction as a consequence of widespread microvascular ischemia. While it is a rare disease (approximately six cases per million per year in the $\mathrm{UK}^{1}$ ), it has a high morbidity and mortality unless it is promptly recognized and timely appropriate therapy initiated. Over the past decade, major advances in the understanding of the underlying pathological basis of TTP have been made, which have led to a more rational approach to designing interventions and have raised the possibility of targeted disease-modifying therapies. In addition, the findings of recently completed prospective trials in patients with TTP are creating a firmer evidence base on which treatment decisions can be made. Despite these recent advances, many aspects of the routine management of TTP are not supported by prospective randomized clinical trials; therefore, significant variation in practice exists. Due to the rarity of this condition and the subsequent lack of familiarity of many clinicians in managing the intense and complex requirements of a patient with TTP, there exists a significant risk of suboptimal outcomes which are potentially avoidable. This review aims to provide a current perspective on the management of TTP as performed by our institution (a TTP referral center in the UK managing approximately $30-40$ new cases of TTP per year), to present the evidence
Correspondence: Marie Scully University College Hospital, 60 Whitfield Street, London WIT 4EU, UK Email m.scully@ucl.ac.uk 
base that guides the current management of TTP, and to discuss potential future directions in therapy.

\section{Pathogenesis and terminology ofTTP}

The underlying pathological mechanism that causes TTP is a severely decreased activity of the metalloprotease ADAMTS13 (a disintegrin and metalloprotease with thrombospondin type 1 repeats, member 13). ${ }^{2,3}$ The in vivo role of ADAMTS13 is to cleave highly hemostatically active ultralarge von Willebrand factor (ULvWF) multimers into smaller protein fragments. $\mathrm{vWF}$ is one of the central proteins of primary hemostasis which, after vessel injury, acts as a bridge between the exposed subendothelial matrix and platelets by binding of the A1 domain of vWF to the GPIb $\alpha$ receptor (in the GPIb/IX/V complex). ULvWF multimers are secreted (both constitutively as well as in response to stress) from endothelial cells and are subsequently cleaved into smaller fragments after exposure of the ADAMTS13 cleavage site (the vWF A2 domain) under the high shear conditions that are typically seen in the arterioles and capillaries. In the presence of severely decreased ADAMTS13 activity, accumulation of excessive amounts of ULvWF multimers bind to platelets in the circulation resulting in platelet aggregation and organ ischemia as a result of partial vessel occlusion by plateletrich thrombi.

Nomenclature regarding TTP is not standardized; this contributes to confusion in the literature, and is a barrier for accurate epidemiological data collection and maintaining disease registries. In our practice, the term "TTP" is restricted to cases with severe deficiency of ADAMTS13 activity $(<10 \%)$. Other thrombotic microangiopathic processes can clinically and pathologically mimic TTP (eg, thrombotic microangiopathy associated with malignancy and atypical hemolytic uremic syndrome); however, while these processes are occasionally associated with decreased ADAMTS13 activity, they are not associated with severe deficiency of ADAMTS13 $(<10 \%)$, so it is preferable to avoid the term TTP in reference to these entities. The severe ADAMTS13 deficiency in TTP is either the result of an autoantibody which binds to ADAMTS13 and impairs its function (approximately $95 \%$ of cases ${ }^{1}$ ), or a congenital deficiency in ADAMTS13 due to mutations present in the ADAMTS13 gene (Figure 1). In the majority of patients with TTP as a result of autoantibody-mediated inhibition of ADAMTS13 ("acquired" TTP), there is no associated clinically overt autoimmune condition or immune dysregulation and the condition is termed primary (or "idiopathic"). In the smaller proportion of patients in which an overt cause of immune dysregulation is identifiable (eg, systemic

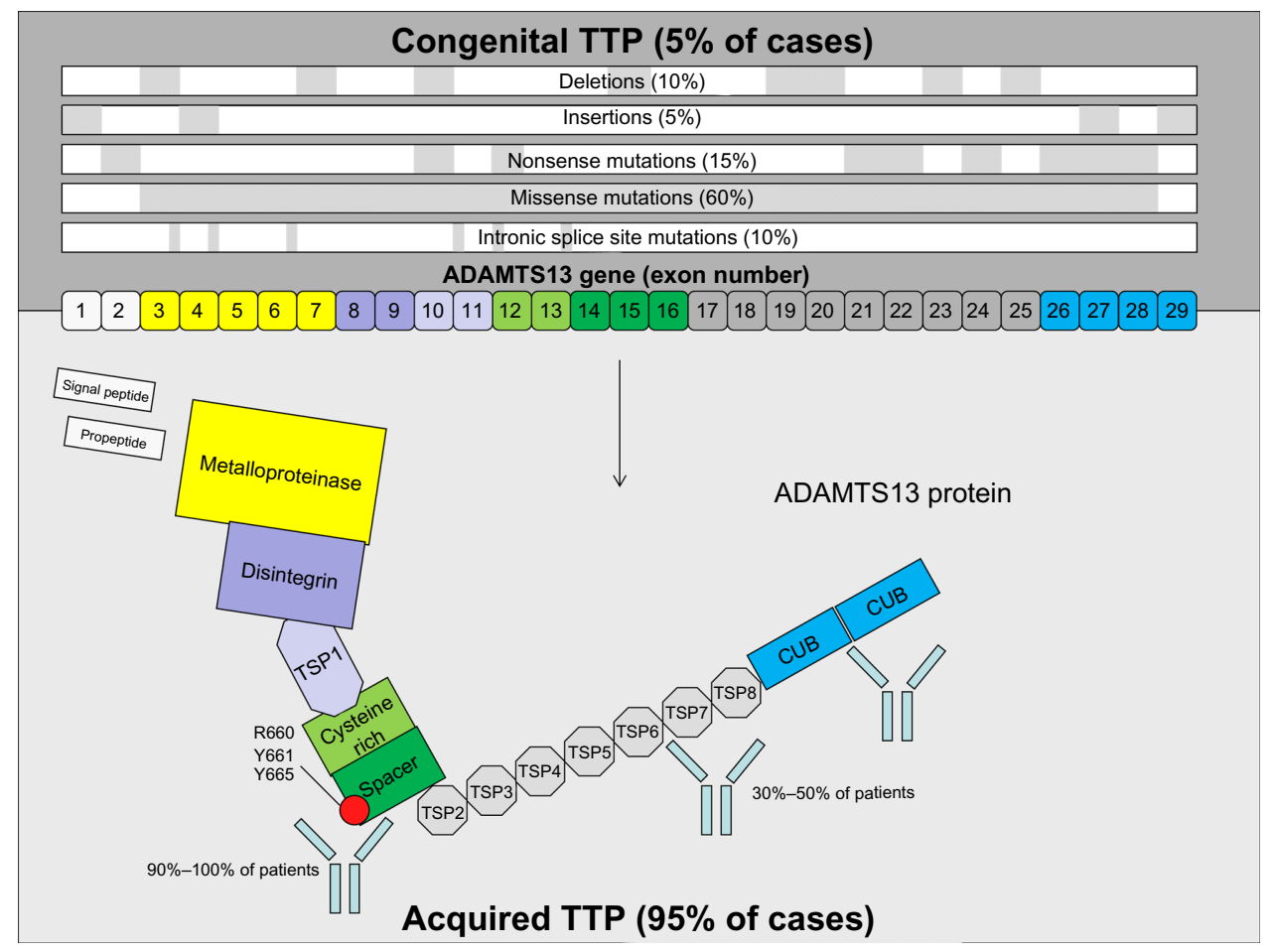

Figure I Genetic mutations and sites of autoantibody specificity in congenital and acquired TTP.

Abbreviations: TTP, thrombotic thrombocytopenic purpura; TSP, thrombospondin-type I repeats; CUB, complement clr/cls, sea Urchin epidermal growth factor, and Bone morphogenetic protein. 
lupus erythematosus, human immunodeficiency virus [HIV] infection), the term "secondary" acquired TTP is used.

The inhibitory autoantibody that is produced in primary or secondary acquired TTP is usually immunoglobulin (Ig)G (and most frequently of the IgG4 subclass) ${ }^{4}$ While antibodies against multiple sites on the ADAMTS13 protein occur in most patients, epitope analysis shows that almost all patients have an antibody directed somewhere between the metalloprotease and spacer domains (in particular Y658-Y665 [R660, Y661, Y665] of the spacer domain) (Figure 1). . $^{5-7}$ This region is crucial for the ADAMTS13 protease function. Congenital TTP is the result of mutations present within the ADAMTS13 gene that result in loss of function of the protein product. ${ }^{8}$ The mutations seen in congenital TTP are heterogenous and consist of a mixture of missense (approximately $60 \%$ ) mutations, nonsense mutations, insertions, and deletions that are distributed throughout the coding region of the gene (Figure 1). ${ }^{8}$ Mutations are usually present in the compound heterozygous state (approximately $65 \%$ of cases), with the remainder occurring as homozygous mutations. ${ }^{8}$ This genetic heterogeneity is reflected in the variable clinical presentation of congenital TTP, including the varied age of onset and severity/frequency of TTP episodes.

\section{Diagnosis of TTP Clinical presentation}

The original clinical description of TTP, combined with subsequent case reports, led to the description of the "classic pentad" of fever, thrombocytopenia, microangiopathic hemolytic anemia, renal dysfunction, and neurological symptoms. ${ }^{9}$ However, based on registry data it is now clear that this pentad is neither sensitive nor specific, and the majority of patients do not have all five clinical features. ${ }^{1}$ In fact, TTP should be suspected, actively excluded by laboratory assays (see below), and empirically treated in the presence of microangiopathic hemolytic anemia (red cell fragmentation on blood film) and thrombocytopenia alone.

The presence of systemic microvascular ischemia in TTP manifests clinically as central nervous system (eg, confusion, visual disturbance, seizure, focal neurological signs [eg, aphasia, hemiparesis] and coma), renal (eg, acute kidney injury), and heart dysfunction (eg, conduction defects, congestive cardiac failure, raised serum troponin). While the dominant cause of "internal" organ dysfunction is ischemia, thrombocytopenia may also lead to "external" bleeding manifestations. Common symptoms and signs include bruising, petechiae, menorrhagia, epistaxis, hematuria, and gastrointestinal hemorrhage. Other clinical features that may be seen in patients with TTP include nonspecific abdominal pain (possibly the result of microvascular ischemia of the gastrointestinal tract), nausea, nonspecific constitutional symptoms, fever, and jaundice (related to hemolysis).

The initial clinical assessment should also aim to identify the presence of secondary causes (eg, pregnancy, autoimmune disease, HIV infection), exclude other microangiopathic processes (eg, malignancy, disseminated intravascular coagulation), and identify potential congenital cases via a thorough family history.

\section{Laboratory assays}

The diagnosis of TTP should be suspected in the presence of the clinical features described above. Given the clinical urgency, treatment should be started empirically before the diagnosis is confirmed with laboratory testing. Despite this, laboratory testing is crucial, to distinguish idiopathic TTP from congenital TTP as well as to differentiate TTP from other microangiopathic processes (eg, malignancy-associated thrombotic microangiopathy and atypical hemolytic uremic syndrome) which have significant implications for treatment.

The laboratory confirmation of TTP is made by the demonstration of a profound decrease in ADAMTS13 enzyme activity (usually $<10 \%$ ) in the plasma of a patient with clinical features of TTP. ADAMTS13 activity can be measured using multiple methodologies; however, the general principle of the assay is the addition of patient plasma to a solution containing vWF (either the entire protein, or more commonly, a shorter peptide containing the ADAMTS13 cleavage site) and subsequently detecting the ADAMTS13-mediated cleavage products. These products can be detected using a variety of endpoints including immunoassay, electrophoresis, or fluorescence resonance energy transfer techniques. Given the heterogeneity of substrate vWF and endpoints used in these assays (as well as other preanalytical and analytical variables), it is important that any ADAMTS13 activity assay that is in diagnostic use is thoroughly validated, with appropriate controls used. While each methodology has specific advantages and disadvantages, ${ }^{10,11}$ our institution uses the fluorescence resonance energy transfer technique to detect cleavage of a recombinant 73 amino acid peptide containing the Y1605-M1606 cleavage site. This assay is relatively rapid to perform (approximately $4-6$ hours per run), allows multiple samples to be run simultaneously, and provides reliable and reproducible results. Results across methodologies are not necessarily reliable; therefore, ideally the same assay should be used for follow-up testing. ${ }^{10}$ 
After a severe decrease in ADAMTS13 activity has been demonstrated, the next step in investigation is the detection of autoantibodies directed against ADAMTS13, thereby confirming the diagnosis of acquired (primary or secondary) TTP. Antibodies to ADAMTS13 can be detected and quantitated using a variety of techniques (eg, immunoassay).

A severe deficiency of ADAMTS13 activity $(<10 \%)$ without a demonstrable autoantibody should raise the suspicion of congenital TTP. Congenital TTP may be subsequently confirmed by sequencing of the ADAMTS13 gene. While there are mutation hotspots within the ADAMTS13 gene, mutations that lead to congenital TTP are distributed throughout the coding region (Figure 1); therefore, comprehensive sequencing of the entire coding region (including splice sites) needs to be performed.

The other investigations that are routinely performed in a patient with newly diagnosed TTP include:

- Blood film, lactate dehydrogenase, haptoglobin, reticulocyte count, bilirubin

- Prothrombin time, activated partial thromboplastin time, fibrinogen

- Liver function tests, urea and electrolytes, serum creatinine, serum troponin, calcium, thyroid function tests

- HIV serology, hepatitis B and C serology

- Pregnancy testing

- Blood group and antibody screen, direct antiglobulin test, cytomegalovirus serology

- Autoantibody screen (eg, antinuclear antibodies, rheumatoid factor, lupus anticoagulant testing)

- CT/MRI brain, echocardiogram, electrocardiogram

- Other imaging and investigations to exclude malignancy as directed by clinical history and examination.

\section{Management of TTP}

\section{Acquired TTP}

The first step in the management of TTP is a high index of suspicion for the diagnosis and timely recognition. Patients with acute TTP display clinical instability and often have sudden, rapid, and unpredictable clinical deterioration. TTP should be actively ruled out in any patient presenting with microangiopathic hemolytic anemia (indicated by red cell fragmentation on blood film) and thrombocytopenia.

Initially, the patient should be resuscitated and any critical organ dysfunction stabilized. Once stable, the site of further management should be decided. The rarity of TTP, combined with the complex and intense clinical needs of this patient population, means that they are ideally managed in centralized institutions experienced in treating patients with TTP.
This treatment plan has numerous potential advantages, including the accumulation of clinical expertise by both clinical hematologists and other medical practitioners (eg, intensive care physicians), the efficient use of on-call apheresis services, standardized ADAMTS13 testing, and an enhanced ability to coordinate clinical trials and maintain disease registries.

Once the diagnosis of TTP is suspected, our practice is to request transfer of the patient to our institution in a timecritical manner. There should be a low threshold for admission to an intensive care unit/high-dependency unit given the intense needs (eg, central venous line placement, plasma exchange), resuscitation required, and clinical instability of these patients within the first 72 hours.

\section{Plasma exchange}

Plasma exchange (PEX) is the most important acute intervention in the management of TTP, and should be initiated without delay in all patients with suspected TTP. Delay in initiation of PEX is a major factor in adverse outcomes ${ }^{12}$ and it is therefore our practice to initiate PEX in patients with TTP transferred to our institution regardless of time of day. PEX is associated with a reduced death rate and is superior to plasma infusion alone, which is the result of the additive effects of both removal of autoantibody by the exchange process (in the case of acquired TTP) as well as supplementing ADAMTS13 activity in the exchanged plasma. ${ }^{13}$ However, plasma infusion is occasionally used as a temporizing measure if there is going to be an unavoidable delay in transfer to our institution from a site without access to PEX.

PEX is initiated at $1.5 \times$ plasma volume $(\mathrm{PV})(60 \mathrm{~mL} / \mathrm{kg})$. In the case of confirmed TTP, PEX at $1.5 \times \mathrm{PV}$ is continued for at least the first 3 days (the regimen utilized in the initial Canadian Apheresis trial in TTP ${ }^{13}$ ), with further PEX dependent on the clinical progress of the patient. It is not unusual for two $1.5 \times \mathrm{PV}$ exchanges to be required per day in the first 72 hours after presentation, and we routinely perform this intensity of PEX in patients with neurological symptoms or evidence of cardiac involvement (eg, raised troponin). One of the considerations in the timing and intensity of PEX over the first few days is the administration of other diseasemodifying therapies (particularly methylprednisolone and rituximab) which may be removed by the PEX procedure. Daily PEX should be continued until 2 days after platelet count normalizes $\left(>150 \times 10^{9} / \mathrm{L}\right)$, and can be safely stopped outright without any weaning schedule. ${ }^{14}$

The process of plasma exchange can either be centrifugation- or membrane-based. When using centrifugation-based therapeutic plasma exchange, blood can either continuously 
flow through the apheresis circuit, or (less frequently) intermittent flow can be used. We use centrifugation-based continuous flow therapeutic plasma exchange for all patients with TTP at our institution.

There are various options for replacement fluid during PEX, including cryosupernatant (CSP), fresh frozen plasma (FFP) or solvent/detergent-treated pooled plasma (SDP). Each of these products has their own advantages and disadvantages. Importantly, they are all safe to use and appear to be of similar efficacy in the treatment of TTP. The lower levels of ULvWF in CSP have historically led to it being viewed as a more attractive option than FFP as a replacement fluid. Indeed, a pilot study appeared to show a benefit of CSP over FFP in patients with TTP. ${ }^{15}$ However, subsequent studies have not reproduced this benefit, so either of these products may be used. ${ }^{16,17}$

SDP (eg, Octaplas, Octapharma AG, Lachen, Switzerland) is produced by pooling plasma from multiple donors who have tested negative for viral markers (according to local regulations specific to site of manufacture) which then undergoes treatment by solvent and detergent, resulting in inactivation (to a 5-6 log reduction) of lipid-enveloped viruses (eg, HIV, hepatitis C). ${ }^{18}$ Decreasing the likelihood of viral transmission is an important consideration given the large amounts of plasma that patients with TTP are exposed to (regularly over $40 \mathrm{~L}$ during initial treatment). In addition, Octaplas is associated with fewer allergic reactions than FFP/CSP as well as a theoretically favorable ULVWF profile (which is similar to CSP). ${ }^{19}$ For these reasons, Octaplas is used for all patients with TTP or using high volumes of plasma in the UK. Plasma products also carry the risk of transfusion-related acute lung injury.

In the case that Octaplas is not available, FFP is usually used due to its general availability. Octaplas is now available with a dedicated prion reduction step by affinity chromatography (OctaplasLG, Octapharma AG) which has been shown to have similar profiles of ADAMTS13 activity and ULVWF to regular SDP. ${ }^{20}$ OctaplasLG has been used in healthy volunteers and appears to be equivalent to Octaplas in regard to clotting factor recovery and safety. ${ }^{21}$ Given its potential enhanced safety, prion-reduced SDP plasma may therefore become the product of choice in the future after its safety and efficacy has been further validated.

\section{Rituximab}

There is sound rationale for the use of the anti-CD20 antibody rituximab in TTP given its demonstrated effectiveness in numerous other antibody-mediated autoimmune disorders. Rituximab is a safe and effective treatment for newly diagnosed TTP, and has been shown in a prospective, multicenter trial to decrease the number of PEX required to achieve remission in Caucasian patients, to decrease length of inpatient stay (in non-intensive care unit patients), and to reduce the risk of relapse by over $80 \%$ when compared to historical controls. ${ }^{22}$ The scheduling of rituximab used in this Phase II trial was adapted from that used in the treatment of lymphoma (ie, $375 \mathrm{mg} / \mathrm{m}^{2}$ intravenously weekly for 4 weeks). ${ }^{22}$ Rituximab treatment may be extended to eight doses in total in those who are slow to respond (ie, with persistently low ADAMTS13 activity or detectable antiADAMTS13 antibodies). ${ }^{22}$

Rituximab is removed from the circulation by PEX; therefore, in the context of daily (or twice daily) PEX, lower trough concentrations of rituximab are achieved. ${ }^{23}$ Importantly, this does not appear to significantly alter the time to B-cell depletion. ${ }^{23}$ Higher trough concentrations are achieved if rituximab is given every 3 to 4 days. ${ }^{23}$ It is our practice to administer rituximab immediately after PEX in order to maximize its duration of action being removed by subsequent PEX. We try and avoid PEX for at least 12 hours after administration of rituximab. If intensive PEX is required due to patient instability, then we administer rituximab more frequently (eg, every 3 days). Intensive initial rituximab scheduling (Day 1, 3, 7, 14) has been used in an open-label prospective French cohort and has been shown to be safe and efficacious. ${ }^{24}$ There is a delay between the administration of rituximab and maximum therapeutic effect on pathological autoantibody production; therefore, it is important to initiate rituximab as soon as possible after the diagnosis of acquired TTP is made. Rituximab administered within 3 days of presentation has been shown in a retrospective study to be associated with more favorable outcomes than if given after 3 days. ${ }^{25}$

In addition to its use in acute TTP, rituximab may be used pre-emptively to prevent relapse of TTP. ${ }^{25,26}$ Any acute episode of TTP is associated with a significant morbidity and mortality; therefore, it is our practice to monitor ADAMTS13 activity and to pre-emptively treat with rituximab in patients who are showing a consistent significant decline in activity. Some patients will have a persistently low ADAMTS13 activity $(<10 \%)$ after clinical resolution of an acute episode of TTP. This emphasizes the fact that TTP is not a single-hit disorder and that inciting events are likely to be required in conjunction with severe ADAMTS13 deficiency in order for a patient to develop the clinical syndrome of acute TTP. We therefore use a combination of the ADAMTS13 activity as well as the clinical context of the patient in order to determine the need for pre-emptive rituximab therapy. 


\section{Corticosteroids}

Steroids are used initially to achieve relatively rapid immunosuppression. There is no firm evidence base to guide the choice of steroid; however, we favor methylprednisolone, as it can be administered intravenously (to avoid absorption issues in critically unwell patients) and has a good safety profile. There is some prospective evidence that higher doses of methylprednisolone $(10 \mathrm{mg} / \mathrm{kg} /$ day $)$ are more effective than lower doses $(1 \mathrm{mg} / \mathrm{kg} /$ day $){ }^{27}$ Our standard practice is to administer methylprednisolone $1 \mathrm{~g}$ intravenously daily for 3 days, with the first dose typically administered immediately after the first PEX.

\section{Supportive care}

PEX, methylprednisolone, and rituximab are the mainstays of the acute management of acquired TTP; however, other important aspects of supportive care include:

- Antiplatelet agents: Low-dose aspirin is commenced in patients with TTP when the platelet count is greater than $50 \times 10^{9} / \mathrm{L}$. Use of aspirin (or dipyridamole) has been associated with a trend to improved early survival. ${ }^{28}$ It is reasonable to use other antiplatelet agents (eg, clopidogrel) in the context of aspirin allergy/intolerance. Our practice is to cease antiplatelet agents on recovery from the initial episode (typically 1-2 months after presentation).

- Antithrombotic therapy: Patients with TTP are usually critically ill and are at high risk of deep venous thrombosis (DVT). We administer pharmacological DVT prophylaxis (typically with low molecular weight heparin) once the platelet count is greater than $50 \times 10^{9} / \mathrm{L}$. Nonpharmacological DVT prophylaxis (eg, graded compression stockings) are useful in the severely thrombocytopenic phase.

- Central venous access: It is often the case that central venous lines need to be inserted in a patient with severe thrombocytopenia. Central venous access is preferable for PEX, and is highly advisable in patients displaying any neurological symptoms or signs of neurological instability. Platelet transfusion is relatively contraindicated in TTP, and timely, effective PEX outweighs the risk of bleeding complications related to line insertion; therefore, central venous catheters should be inserted regardless of platelet count. We favor internal jugular vein catheterization due to a lower risk of infection, less restriction on patient mobility, and improved apheresis performance. In severe thrombocytopenia (eg, platelet count $<20 \times 10^{9} / \mathrm{L}$ ), it is reasonable to use the femoral route due to the perceived enhanced safety of this approach. Due to the higher risk of the procedure, it is vital that the operator has sufficient expertise in central line insertion in order for the procedure to be performed quickly and as atraumatically as possible. It is also important that indwelling venous access is changed regularly, as line infection is not uncommon; it can often drive the underlying disease process, and is a common cause of suboptimal response to PEX. In the absence of signs of infection, femoral lines are changed every 48 hours, and internal jugular lines are changed every 7 days.

- Hepatitis B prophylaxis: Patients who have prior hepatitis B exposure (regardless of the current carrier status) are at risk for potentially fatal hepatitis B reactivation in the context of B-cell depletion with rituximab therapy. Local guidelines should be followed in regard to antiviral agents and durations (eg, lamivudine $100 \mathrm{mg}$ orally daily for 6 months after last rituximab exposure).

- Folic acid: Due to the hemolytic state, folic acid supplementation (eg, $5 \mathrm{mg}$ orally daily) should be given to all patients.

\section{Other interventions}

There are numerous interventions that have been used historically in patients with TTP which have a limited role in the contemporary management of the disorder. These include:

- Other immunosuppressive medication: While specific B-cell modulation with rituximab is by far the most effective immunomodulation in TTP, in the context of refractory disease despite adequate rituximab therapy (or true rituximab intolerance), second-line immunosuppression may be used. Agents used in this context include mycophenolate mofetil, cyclosporine, and vincristine. None of these agents have high-quality data regarding their efficacy (especially in the context of rituximab treatment).

- Splenectomy: The role of splenectomy in the current management of TTP is limited. Historically, it has been performed for relapsing disease; however, there is little good evidence regarding its efficacy and safety. Rituximab should be considered the mainstay of treatment for relapsing disease.

\section{Congenital TTP}

The pathogenic basis of congenital TTP is decreased ADAMTS13 activity secondary to deleterious mutations within the ADAMTS13 gene. Autoantibodies against ADAMTS13 do not occur in congenital TTP; therefore, immunosuppression (eg, with methylprednisolone or rituximab) is not indicated. Acute episodes of TTP in patients 
with known congenital TTP are treated with PEX/plasma infusion alone in a similar way to acquired TTP. Once in remission, subsequent treatment depends on the phenotype of the individual patient. Some patients with congenital TTP require plasma infusions every 3-4 weeks in order to replace functional ADAMTS13 and prevent symptomatic TTP episodes, whereas others will only require treatment in the context of inciting factors like pregnancy or infection. The amount of plasma infused is usually one therapeutic dose (eg, 10-15 mL/kg of FFP/SDP). In prophylactic schedules, the interval between plasma infusions is empirically determined, and there is no evidence to support a particular target ADAMTS13 activity.

Patients with congenital TTP have a significant lifetime exposure to plasma; therefore, the safest product in regard to viral/prion transmission should be used. It is our practice to use SDP with prion removal (eg, OctaplasLG) for replacement therapy in congenital TTP.

\section{Special circumstances Pregnancy}

TTP may present during pregnancy, and it needs to be differentiated from other microangiopathic processes that can occur (pre-eclampsia, HELLP [hemolysis, elevated liver enzymes, low platelets] syndrome, disseminated intravascular coagulation). TTP in pregnancy may represent one of two processes: either acquired TTP due to the changes in immune regulation that occur during pregnancy, or precipitation of an episode of symptomatic TTP in a patient with congenital TTP (which may or may not have been previously recognized).

Acute TTP in pregnancy has a significant maternal/fetal morbidity and mortality and requires aggressive treatment. In the case of acquired TTP with a demonstrated autoantibody, the patient should be treated with methylprednisolone and PEX in a similar way to nonpregnant patients. Rituximab is not advocated during pregnancy due to unknown risks to the developing fetus. Management of the pregnant woman is dependent on the stage of pregnancy and the presence of fetal compromise. In general, women are delivered as soon as it is safe to do so from a maternal/fetal point of view. It should be noted that delivery of the baby does not necessarily result in the resolution of the TTP episode.

Relapse of TTP is a risk in patients that have had a previous episode of acquired TTP that become pregnant. We actively monitor pregnant women that have had a previous TTP episode with frequent ADAMTS13 levels throughout pregnancy and intervene with steroids with or without azathioprine if a consistent drop in ADAMTS13 activity is seen.
PEX can be performed during pregnancy and is utilized for severe decreases in ADAMTS13 activity and symptomatic TTP. Patients with congenital TTP can generally be managed with plasma infusions alone, which are necessary throughout pregnancy, starting in the first trimester.

\section{HIV-associated TTP}

HIV infection is an important cause of secondary TTP. Achieving adequate virological suppression is an important part of therapy in HIV-associated TTP. Patients with higher viral loads at presentation $(\mathrm{eg},>500,000 \mathrm{copies} / \mathrm{mL})$ require more PEX to achieve remission. ${ }^{29}$ Importantly, when combined with highly active antiretroviral therapy, PEX and corticosteroid alone (without rituximab) is usually sufficient to achieve a remission from the acute episode of TTP. $^{29}$ If consistent viral suppression can be achieved, the risk of relapse (even without rituximab) is low. Conversely, loss of virological response (either from nonadherence or virus resistance) can precipitate acute episodes of symptomatic TTP.

\section{Future directions}

While the outcomes of patients with TTP have improved over time, there is still significant potential for the development of new agents that target various aspects of the disease process, including:

- Recombinant ADAMTS13: The availability of a recombinant form of ADAMTS13 would mean the ability to achieve higher ADAMTS13 activity than is currently possible by transfusing donor plasma. In vitro, recombinant ADAMTS13 is able to overcome neutralizing autoantibodies and result in the reconstitution of ADAMTS13 activity in plasma samples from patients with TTP. ${ }^{30}$ Moreover, modification of ADAMTS13 by altering amino acids in exosite 3 in the spacer domain can result in an ADAMTS13 protein that is both resistant to the majority of autoantibodies from patients with TTP and shows enhanced enzymatic activity. ${ }^{31}$

- Anti-vWF nanobody (ALX-0081/ALX-0681): Currently being evaluated in a Phase II trial in patients with acquired TTP,ALX-0081/ALX-0681 is a bivalent nanobody (a therapeutic protein derived from the heavy chain variable region of heavy chain only immunoglobulins) that acts by binding to the N-terminus of the vWF A1 domain and subsequently blocking its interaction with platelets. ${ }^{32}$ It has been shown to be effective in animal models of TTP thus far and to have an acceptable safety profile in humans. ${ }^{33}$ Targeting of the vWF A1-domain-platelet interaction has also been trialed 
with the aptamer (nucleic acid macromolecule) ARC1779. Although this trial was prematurely closed due to financial reasons, ARC1779 showed an encouraging safety profile and warrants further evaluation in prospective randomized trials. ${ }^{34}$

- N-acetyl cysteine: $\mathrm{N}$-acetyl cysteine has multiple effects on VWF in vitro that may be beneficial in TTP, including reduction in vWF multimer size as well as inhibition of platelet aggregation. ${ }^{35}$ Its use in patients with TTP is currently being evaluated.

- Novel immunosuppressive agents: Given the effectiveness of rituximab in acquired TTP, it is reasonable to suggest that other B-cell targeted immunotherapy (eg, second-generation anti-CD20 antibodies) may be of potential value. Other B-cell directed therapies, such as Bruton tyrosine kinase inhibitors (which have shown activity in animal models of autoimmune disease ${ }^{36,37}$ ) may also merit evaluation. In addition to B-cell directed therapy, there have been two case reports of the proteasome inhibitor bortezomib being used in cases of rituximab-refractory TTP with reported efficacy. ${ }^{38,39}$

\section{Conclusion}

The last decade has seen significant advances in the understanding of the pathological basis of TTP. This has resulted in improvement in outcomes through the use of therapies that are rationally targeted at the disease biology. Despite these improvements, TTP still has a high mortality rate, and there is significant room for improvement in numerous areas of patient care. We advocate the centralized management of patients with TTP in order to concentrate expertise and experience in this rare condition. In addition to the treatment benefits, this will allow more effective participation and coordination of clinical trials.

\section{Disclosure}

The authors report no conflicts of interest in this work.

\section{References}

1. Scully M, Yarranton H, Liesner R, et al. Regional UK TTP registry: correlation with laboratory ADAMTS 13 analysis and clinical features. Br J Haematol. 2008;142(5):819-826.

2. Furlan M, Robles R, Galbusera M, et al. von Willebrand factor-cleaving protease in thrombotic thrombocytopenic purpura and the hemolyticuremic syndrome. N Engl J Med. 1998;339(22):1578-1584.

3. Fujikawa K, Suzuki H, McMullen B, Chung D. Purification of human von Willebrand factor-cleaving protease and its identification as a new member of the metalloproteinase family. Blood. 2001;98(6):1662-1666.

4. Ferrari S, Mudde GC, Rieger M, Veyradier A, Kremer Hovinga JA, Scheiflinger F. IgG subclass distribution of anti-ADAMTS13 antibodies in patients with acquired thrombotic thrombocytopenic purpura. J Thromb Haemost. 2009;7(10):1703-1710.
5. Yamaguchi Y, Moriki T, Igari A, et al. Epitope analysis of autoantibodies to ADAMTS13 in patients with acquired thrombotic thrombocytopenic purpura. Thromb Res. 2011;128(2):169-173.

6. Klaus C, Plaimauer B, Studt JD, et al. Epitope mapping of ADAMTS13 autoantibodies in acquired thrombotic thrombocytopenic purpura. Blood. 2004;103(12):4514-4519.

7. Pos W, Crawley JT, Fijnheer R, Voorberg J, Lane DA, Luken BM. An autoantibody epitope comprising residues R660, Y661, and Y665 in the ADAMTS13 spacer domain identifies a binding site for the A2 domain of VWF. Blood. 2010;115(8):1640-1649.

8. Lotta LA, Garagiola I, Palla R, Cairo A, Peyvandi F. ADAMTS13 mutations and polymorphisms in congenital thrombotic thrombocytopenic purpura. Hum Mutat. 2010;31(1):11-19.

9. Moschcowitz E. An Acute Febrile Pleiochromic Anemia with Hyaline Thrombosis of the Terminal Arterioles and Capillaries - an undescribed disease. Arch Int Med. 1925;36:89-93.

10. Mackie I, Langley K, ChitolieA, et al. Discrepancies between ADAMTS13 activity assays in patients with thrombotic microangiopathies. Thromb Haemost. 2013;109(3):488-496.

11. Starke R, Machin S, Scully M, Purdy G, Mackie I. The clinical utility of ADAMTS13 activity, antigen and autoantibody assays in thrombotic thrombocytopenic purpura. Br J Haematol. 2007;136(4):649-655.

12. Pereira A, Mazzara R, Monteagudo J, et al. Thrombotic thrombocytopenic purpura/hemolytic uremic syndrome: a multivariate analysis of factors predicting the response to plasma exchange. Ann Hematol. 1995;70(6): 319-323.

13. Rock GA, Shumak KH, Buskard NA, et al. Comparison of plasma exchange with plasma infusion in the treatment of thrombotic thrombocytopenic purpura. Canadian Apheresis Study Group. N Engl J Med. 1991;325(6):393-397.

14. Bandarenko N, Brecher ME. United States Thrombotic Thrombocytopenic Purpura Apheresis Study Group (US TTP ASG): multicenter survey and retrospective analysis of current efficacy of therapeutic plasma exchange. J Clin Apher. 1998;13(3):133-141.

15. Rock G, Shumak KH, Sutton DM, Buskard NA, Nair RC. Cryosupernatant as replacement fluid for plasma exchange in thrombotic thrombocytopenic purpura. Members of the Canadian Apheresis Group. Br J Haematol. 1996;94(2):383-386.

16. Zeigler ZR, Shadduck RK, Gryn JF, et al; North American TTP Group. Cryoprecipitate poor plasma does not improve early response in primary adult thrombotic thrombocytopenic purpura (TTP). J Clin Apher. 2001;16(1):19-22.

17. Rock G, Anderson D, Clark W, et al; Canadian Apheresis Group; Canadian Association of Apheresis Nurses. Does cryosupernatant plasma improve outcome in thrombotic thrombocytopenic purpura? No answer yet. Br J Haematol. 2005;129(1):79-86.

18. Hellstern P, Solheim BG. The Use of Solvent/Detergent Treatment in Pathogen Reduction of Plasma. Transfus Med Hemother. 2011;38(1): 65-70.

19. Scully M, Longair I, Flynn M, Berryman J, Machin SJ. Cryosupernatant and solvent detergent fresh-frozen plasma (Octaplas) usage at a single centre in acute thrombotic thrombocytopenic purpura. Vox Sang. 2007;93(2):154-158.

20. Lawrie AS, Green L, Canciani MT, et al. The effect of prion reduction in solvent/detergent-treated plasma on haemostatic variables. Vox Sang. 2010;99(3):232-238.

21. Jilma-Stohlawetz P, Kursten FW, Horvath M, et al. Recovery, safety, and tolerability of a solvent/detergent-treated and prion-safeguarded transfusion plasma in a randomized, crossover, clinical trial in healthy volunteers. Transfusion. 2013;53(9):1906-1917.

22. Scully M, McDonald V, Cavenagh J, et al. A phase 2 study of the safety and efficacy of rituximab with plasma exchange in acute acquired thrombotic thrombocytopenic purpura. Blood. 2011;118(7):1746-1753.

23. McDonald V, Manns K, Mackie IJ, Machin SJ, Scully MA. Rituximab pharmacokinetics during the management of acute idiopathic thrombotic thrombocytopenic purpura. J Thromb Haemost. 2010;8(6): $1201-1208$. 
24. Froissart A, Buffet M, Veyradier A, et al; French Thrombotic Microangiopathies Reference Center. Efficacy and safety of first-line rituximab in severe, acquired thrombotic thrombocytopenic purpura with a suboptimal response to plasma exchange. Experience of the French Thrombotic Microangiopathies Reference Center. Crit Care Med. 2012;40(1):104-111.

25. Westwood JP, Webster H, McGuckin S, McDonald V, Machin SJ, Scully M. Rituximab for thrombotic thrombocytopenic purpura: benefit of early administration during acute episodes and use of prophylaxis to prevent relapse. J Thromb Haemost. 2013;11(3):481-490.

26. Bresin E, Gastoldi S, Daina E, et al. Rituximab as pre-emptive treatment in patients with thrombotic thrombocytopenic purpura and evidence of anti-ADAMTS13 autoantibodies. Thromb Haemost. 2009;101(2):233-238.

27. Balduini CL, Gugliotta L, Luppi M, et al; Italian TTP Study Group. High versus standard dose methylprednisolone in the acute phase of idiopathic thrombotic thrombocytopenic purpura: a randomized study. Ann Hematol. 2010;89(6):591-596.

28. Bobbio-Pallavicini E, Gugliotta L, Centurioni R, et al. Antiplatelet agents in thrombotic thrombocytopenic purpura (TTP). Results of a randomized multicenter trial by the Italian Cooperative Group for TTP. Haematologica. 1997;82(4):429-435.

29. Hart D, Sayer R, Miller R, et al. Human immunodeficiency virus associated thrombotic thrombocytopenic purpura - favourable outcome with plasma exchange and prompt initiation of highly active antiretroviral therapy. Br J Haematol. 2011;153(4):515-519.

30. Plaimauer B, Kremer Hovinga JA, Juno C, et al. Recombinant ADAMTS13 normalizes von Willebrand factor-cleaving activity in plasma of acquired TTP patients by overriding inhibitory antibodies. J Thromb Haemost. 2011;9(5):936-944.

31. Jian C, Xiao J, Gong L, et al. Gain-of-function ADAMTS13 variants that are resistant to autoantibodies against ADAMTS13 in patients with acquired thrombotic thrombocytopenic purpura. Blood. 2012;119(16) 3836-3843.
32. Ulrichts H, Silence K, Schoolmeester A, et al. Antithrombotic drug candidate ALX-0081 shows superior preclinical efficacy and safety compared with currently marketed antiplatelet drugs. Blood. 2011;118(3):757-765.

33. Callewaert F, Roodt J, Ulrichts H, et al. Evaluation of efficacy and safety of the anti-VWF Nanobody ALX-0681 in a preclinical baboon model of acquired thrombotic thrombocytopenic purpura. Blood. 2012;120(17): 3603-3610.

34. Cataland SR, Peyvandi F, Mannucci PM, et al. Initial experience from a double-blind, placebo-controlled, clinical outcome study of ARC1779 in patients with thrombotic thrombocytopenic purpura. Am J Hematol. 2012;87(4):430-432.

35. Chen J, Reheman A, Gushiken FC, et al. N-acetylcysteine reduces the size and activity of von Willebrand factor in human plasma and mice. J Clin Invest. 2011;121(2):593-603.

36. Chang BY, Huang MM, Francesco M, et al. The Bruton tyrosine kinase inhibitor PCI-32765 ameliorates autoimmune arthritis by inhibition of multiple effector cells. Arthritis Res Ther. 2011;13(4):R115.

37. Honigberg LA, Smith AM, Sirisawad M, et al. The Bruton tyrosine kinase inhibitor PCI-32765 blocks B-cell activation and is efficacious in models of autoimmune disease and B-cell malignancy. Proc Natl Acad Sci U S A. 2010;107(29):13075-13080.

38. Shortt J, Oh DH, Opat SS. ADAMTS13 antibody depletion by bortezomib in thrombotic thrombocytopenic purpura. $N$ Engl J Med 2013;368(1):90-92.

39. van Balen T, Schreuder MF, de Jong H, van de Kar NC. Refractory thrombotic thrombocytopenic purpura in a 16-year-old girl: successful treatment with bortezomib. Eur J Haematol. 2014;92(1):80-82.

\section{Publish your work in this journal}

The Journal of Blood Medicine is an international, peer-reviewed, open access, online journal publishing laboratory, experimental and clinical aspects of all topics pertaining to blood based medicine including but not limited to: Transfusion Medicine; Blood collection, Donor issues, Transmittable diseases, and Blood banking logistics; Immunohematology; Artificial and alternative

\section{Dovepress}

blood based therapeutics; Hematology; Biotechnology/nanotechnology of blood related medicine; Legal aspects of blood medicine; Historical perspectives. The manuscript management system is completely online and includes a very quick and fair peer-review system. Visit http://www.dovepress.com/ testimonials.php to read real quotes from published authors. 